\title{
Corrigendum: Tripropeptins, novel antimicrobial agents produced by Lysobacter sp. II. structure elucidation
}

\author{
Hideki Hashizume, Sehei Hirosawa, Ryuichi Sawa, Yasuhiko Muraoka, Daishiro Ikeda, Hiroshi Naganawa \\ and Masayuki Igarashi
}

The Journal of Antibiotics (2016) 69, 889-891; doi:10.1038/ja.2016.104

Correction to: The Journal of Antibiotics (2004) 57, 52-58; doi:org/10.7164/antibiotics.57.52

The authors of the above article did misjudgment the stereochemistry of tripropeptins-constituent serine residue which was determined by Marfey's method using HPLC. From our recent analysis using advanced Marfey's method (LC/MS) revealed that the stereochemistry of serine residue was proved to be D-form as shown below. Resulting chromatograms and the conditions were shown in Supplementary Figure S1.

Adding to above, we corrected the Figures 1 and 3 as follows. Authors would like to apologize for the mistakes.



Figure S1 Extracted ion chromatograms of L-FDLA derivatives of (A) TPPC hydrolysate and (B) authentic serines. Acid hydrolysate of tripropeptin C (TPPC) and authentic serines were coupled with $N^{\alpha}$-(5-fluoro-2,4-dinitrophenyl)-L-leucinamide (FDLA). Resulting samples were subjected to LC-MS analysis. Extracted ion chromatograms, monitoring at $\mathrm{m} / \mathrm{z}$ 400.14-400.15 (FDLA coupled serine) were shown. HPLC conditions of Supplementary Figure S1: Device: LTQ Orbitrap (Thermo Fisher Scientific, Waltham, MA). Column: Shiseido Capcell Pak UG120, 2×150 mm, $5 \mu \mathrm{m}$. Solvent system: 0-20 min, 20\%A-60\%A linear gradient. A: $\mathrm{CH}_{3} \mathrm{CN}-0.01 \% \mathrm{HCOOH}, \mathrm{B}: 0.01 \% \mathrm{HCOOH}$. Flow rate: $0.3 \mathrm{ml} \mathrm{min}{ }^{-1}$. Temperature: $50^{\circ} \mathrm{C}$. A full color version of this figure is available at The Journal of Antibiotics online. 


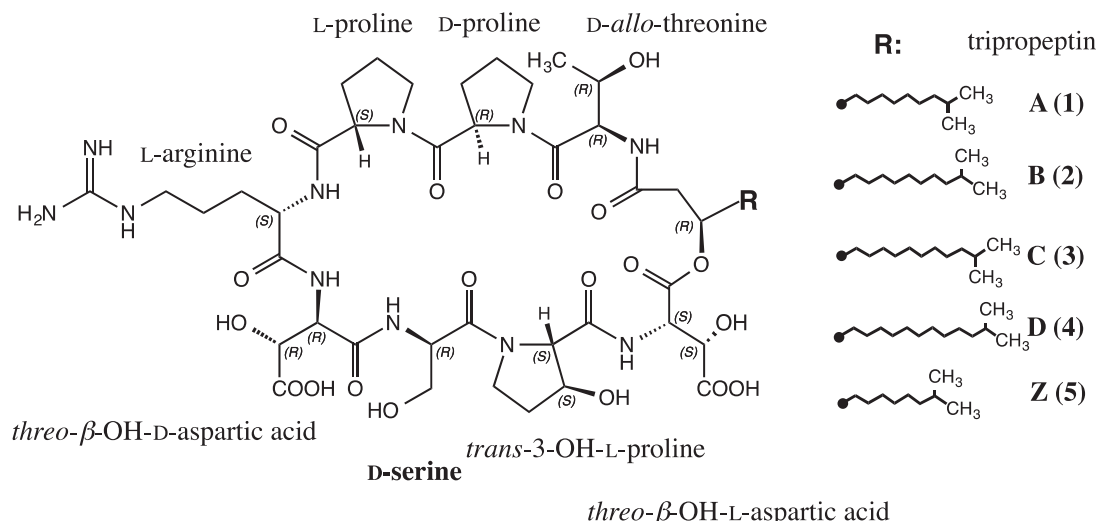

Figure 1 Structure of tripropeptins. 

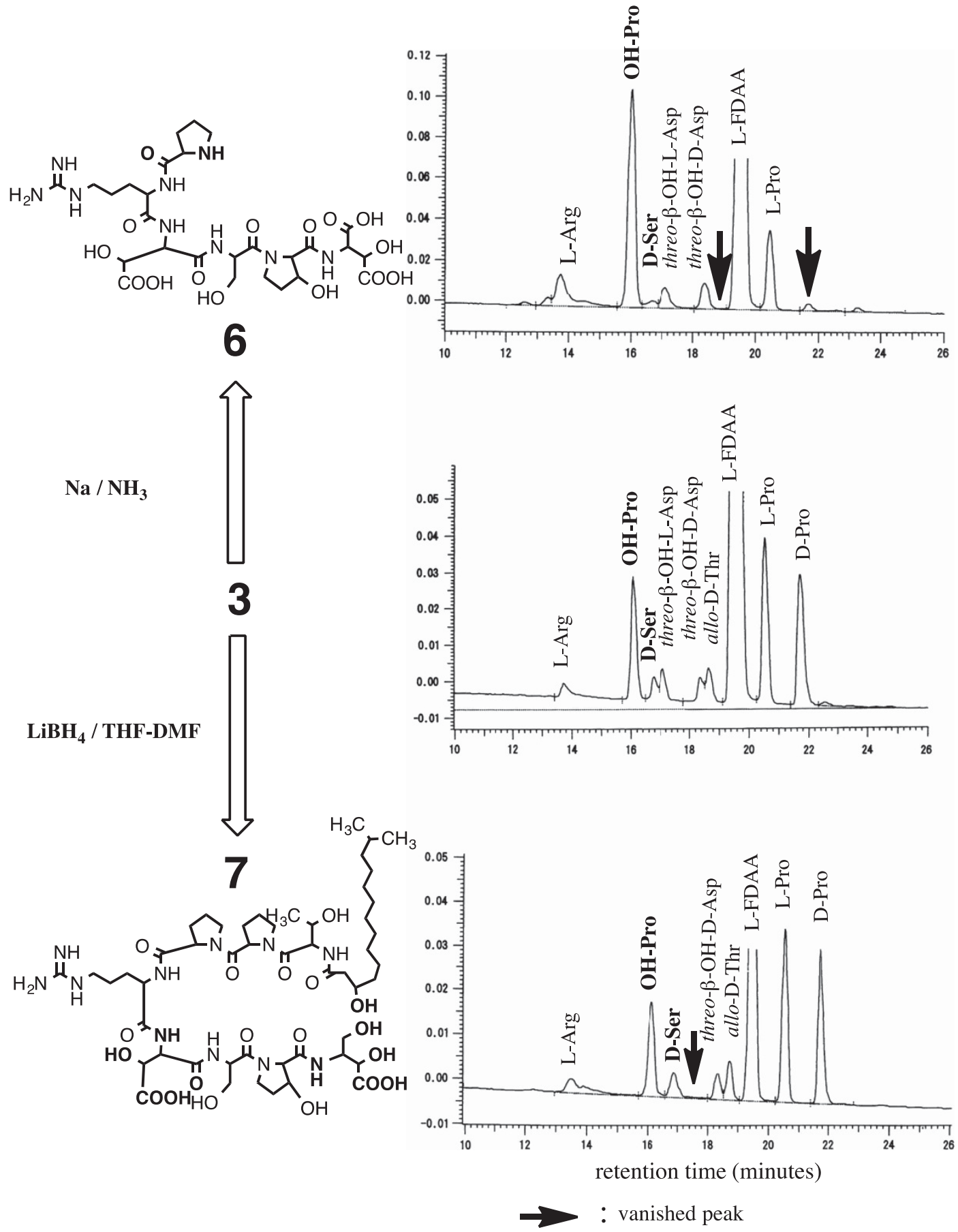

Figure 3 Amino acid analysis of the fragments 6 and 7 . 